\title{
Cultivation and identification of rat bone marrow-derived mesenchymal stem cells
}

\author{
KE SONG, MENGQI HUANG, QI SHI, TIANFENG DU and YINGGUANG CAO \\ Department of Prosthodontics and Implantology, Tongji Hospital, Tongji Medical College, \\ Huazhong University of Science and Technology, Wuhan, Hubei 430030, P.R. China
}

Received September 1, 2013; Accepted April 25, 2014

DOI: $10.3892 / \mathrm{mmr} .2014 .2264$

\begin{abstract}
Bone marrow-derived mesenchymal stem cells (BMSCs) have the potential to form a variety of mesenchymal tissue types, which are a source of cells for bone tissue engineering applications. The present study attempted to establish an effective and convenient method for culturing BMSCs. Total bone marrow cells, which were harvested from rat femurs, were cultured and BMSCs were selected and expanded through passaging in vitro. Furthermore, the biological properties of BMSCs were investigated, specific surface antigen expression was assessed using flow cytometry and the multipotent differentiation potential characteristics were demonstrated using standard in vitro conditions. Monoptychial heterogeneous cells were obtained. A total of $98.4 \%$ of cells at passage 3 expressed cluster of differentiation (CD)29 and CD90, but not CD45. The cells were able to differentiate into osteogenic and adipogenic cells. In conclusion, BMSCs that are isolated from the rat bone marrow and exhibit the identified characteristics may be used as seed cells in bone tissue engineering.
\end{abstract}

\section{Introduction}

The destruction of alveolar and jaw bone, often a consequence of trauma, tumor or periodontitis, may have a direct impact on the stability of mouth rehabilitation therapy and limit the use of dental implants. Bone grafting is required in the fields of reconstructive, orthopedic and craniofacial surgery, as well as dental implantology (1). Autologous or allogenic bone grafting has been applied for these pathological conditions. However, a lack of sufficient materials precludes the use of autologous bone while the use of allogenic bone for transplantation carries

Correspondence to: Dr Yingguang Cao, Department of Prosthodontics and Implantology, Tongji Hospital, Tongji Medical College, Huazhong University of Science and Technology, 1095 Jiefang Avenue, Wuhan, Hubei 430030, P.R. China

E-mail: cyg0729@tjh.tjmu.edu.cn

Key words: bone marrow-derived mesenchymal stem cells, bone tissue engineering, biological properties a potential risk of immune responses. Progress in materials science and biology has resulted in the possibility of bone tissue engineering with the aim of producing a bony equivalent in vitro by combining bone forming cells and a synthetic three-dimensional scaffold (2-4).

Bone marrow-derived mesenchymal stem cells (BMSCs) have the potential to form a variety of mesenchymal tissue types, including bone, cartilage, tendon, ligament, muscle and fat (5-9). BMSCs can be isolated and cultured to large numbers from a small volume of bone marrow, and are therefore sources of cells for bone tissue engineering applications.

The number of studies on BMSCs has increased markedly over the last two decades, reflecting a rising biological and clinical interest in these cells. BMSCs are attracting focus not only from established laboratories, but also from scientists that are new to the field. As such, scientific understanding is likely to be rapidly enhanced, leading to the accelerated development of novel cellular therapies (10). However, this increasing interest has also resulted in numerous ambiguities and reports of contradictory data.

There is no general consensus as to the defining characteristics of BMSCs. Numerous laboratories have developed techniques to isolate and expand BMSCs exhibiting apparently similar properties from a number of tissue types (10). However, the variations in the tissue sources and methodologies used for the cell preparation may mean that it is not possible to directly compare the reported biological properties and experimental findings, particularly in the context of cell therapy, since the cells may exhibit insufficient similarities. The uncertainty, with regard to cell equivalence, is, in part, due to the lack of universally accepted criteria to define BMSCs. Significantly, the inability to compare and contrast studies from different groups is likely to hinder progress in the field.

The present study attempted to determine an effective and convenient method for culturing BMSCs in order to produce osteogenic seeding cells for bone tissue engineering. Total bone marrow cells, which were harvested from rat femurs, were cultured and BMSCs were selected and expanded through passaging in vitro. Furthermore, the biological properties of BMSCs were investigated, specific surface antigen (Ag) expression was assessed using flow cytometry and the multipotent differentiation potential characteristics of the cells were demonstrated using standard in vitro conditions. In addition, the osteogenic potential of rat BMSCs in several passages was 
evaluated by assessing mRNA expression of osteogenic factors in the cultured cells.

\section{Materials and methods}

Materials. Low-glucose Dulbecco's modified Eagle's medium (L-DMEM) was purchased from Invitrogen Life Technologies (Gibco; Carlsbad, CA, USA). L-glutamine, penicillin/streptomycin antibiotics (AB), trypsin/EDTA and phosphate-buffered saline (PBS) were purchased from Sigma-Aldrich (St. Louis, MO, USA). Fetal bovine serum (FBS) was supplied by Thermo Fisher Scientific Inc. (Waltham, MA, USA). Fluorescein isothiocyanate (FITC) anti-mouse/rat cluster of differentiation (CD)29, phycoerythrin (PE) anti-rat CD90 and allophycocyanin (APC) anti-rat CD45 were purchased from BioLegend (San Diego, CA, USA). Sprague Dawley (SD) rat mesenchymal stem cell osteogenic differentiation medium and SD rat mesenchymal stem cell adipogenic differentiation medium were purchased from Cyagen Biosciences Inc. (Santa Clara, CA, USA). The alkaline phosphatase (ALP) detection kit and ALP staining solution were supplied by Nanjing Jiancheng Bioengineering Institute (Nanjing, China). All other chemicals were from standard laboratory suppliers and were of the highest purity available. The present study was approved by the ethics committee of Huazhong University of Science and Technology (Wuhan, China).

Cell isolation and culture. SD rats (specific pathogen free, male, three weeks old) were purchased from a professional breeder (Vital River, Beijing, China). The animal study was approved by the local committee for Animal Care and Ethics. The rats were sacrificed, the pelt was wetted thoroughly with $70 \%$ isopropanol and the hind limbs were clipped and peeled. The knee joint in the center was cut using sterile sharp scissors, and the ligaments and excess tissue were removed. At the same time, the femur and tibia were severed at the hip and ankle, respectively. The surrounding muscles, ligaments and excess tissue were detached from the bone. The ends of the long bones were trimmed to expose the interior of the marrow shaft. Both femoral epiphyses were cut and the medulla was carefully flushed with $3 \mathrm{ml}$ L-DMEM containing 10\% FBS and 1\% AB using a syringe with an 18-gauge needle. Using the same needle and syringe on ice, medium and cells were gently drawn up and down several times to generate a single-cell suspension. The bone marrow suspensions were cultured in polystyrene six-well dishes and non-adherent cells were removed from the culture after two days by a series of washes in PBS and subsequent changes of medium. Adherent cells were expanded as monolayer cultures in $5 \% \mathrm{CO}_{2} / 95 \%$ air atmosphere at $37^{\circ} \mathrm{C}$ with the medium being exchanged every three days. These primary cells were referred to as passage $0(\mathrm{P} 0)$. The confluent cells were dissociated with $0.25 \%$ trypsin and $0.01 \%$ EDTA, and subcultured in new six-well culture dishes at a plating density of $5 \times 10^{4}$ cells/well. These procedures were repeated four times and the cultures were referred to as P1, P2, P3, P4 and P5.

Cell viability assay. The inhibition of cell proliferation by genistein was assessed using MTT assays which monitor the number of viable cells based on the reduction of MTT by the mitochondrial dehydrogenases. BMSCs at P3 were plated into 96-well tissue culture dishes at an initial density of 600 cells/well in $200 \mu \mathrm{l}$ medium. Following incubation for $24 \mathrm{~h}, 20 \mu \mathrm{l}$ MTT reagent ( $5 \mathrm{mg} / \mathrm{ml}$; Sigma-Aldrich) was added to each well and, subsequent to further incubation at $37^{\circ} \mathrm{C}$ for $4 \mathrm{~h}$, the supernatant was removed and the formazan crystals were dissolved by adding $150 \mu \mathrm{l}$ dimethylsulfoxide. The plate was then read on a microplate reader at $490 \mathrm{~nm}$. Experiments were conducted in triplicate. The results were expressed as relative MTT activity as compared with the control conditions (blank well on plastic).

Cell cycle assay. P3 cells were typsinized, washed with PBS and fixed with $70 \%$ ethanol. The fixed cells were centrifuged at $1,000 \mathrm{~g}$ for $5 \mathrm{~min}$ and resuspended in PBS at a concentration of $1 \times 10^{6}$ cells $/ \mathrm{ml}$. Following incubation with $10 \mu \mathrm{l}$ ribonuclease A (RNase A), $10 \mu \mathrm{l}$ propidium iodide (PI; $500 \mu \mathrm{g} / \mathrm{ml}$ ) at room temperature for $30 \mathrm{~min}$, the cell suspension was quantified using flow cytometry.

Specific surface Ag expression assay. To determine the phenotypic expression of stem cell markers in cultured BMSCs, flow cytometry was performed. Cultured P3 cells were harvested and washed twice in PBS. Cells were then stained with FITC-conjugated mouse anti-rat CD29 (BioLegend), PE-conjugated rabbit anti-rat CD90 (BioLegend) and APC-conjugated rabbit anti-rat CD45 (BioLegend) antibodies (diluted 1:100), washed twice in PBS and analyzed using flow cytometry. At least 10,000 events were collected and further analyzed with CellQuest V3.3 (Becton-Dickinson, Franklin Lakes, NJ, USA). In addition, a triple immunofluorescence technique was performed to identify the specific surface $\mathrm{Ag}$ expression of BMSCs, and a confocal laser scanning microscope was used to observe the staining.

Osteogenic differentiation assays. $\mathrm{P} 3$ cells were replated in growth medium at $3 \times 10^{3}$ cells $/ \mathrm{cm}^{2}$ in six-well tissue culture plates. Following incubation for $24 \mathrm{~h}$, the growth medium was replaced with OriCell ${ }^{\mathrm{TM}}$ SD rat mesenchymal stem cell osteogenic differentiation medium (Cyagen Biosciences, Inc.). Cells were then cultured in a $5 \% \mathrm{CO}_{2} / 95 \%$ air atmosphere at $37^{\circ} \mathrm{C}$ with the osteogenic differentiation medium being replaced every three days.

After 3, 5, 7, 9, 11 and 13 days, the supernatants were collected into 96-well tissue culture dishes and the BMSCs were suspended in $100 \mu 1 \mathrm{PBS}$, respectively. The ALP activity in the supernatant medium secreted by the BMSCs was quantified using an ALP assay kit (Nanjing Jiancheng Bioengineering Institute). The 96-well plates were then read on a microplate reader at $520 \mathrm{~nm}$. The absorbance was used to calculate the protein concentration and expressed as King Armstrong units. In addition, the cell/PBS suspension was sonicated on ice for $30 \mathrm{sec}$ using an ultrasonic homogenizer (Cole-Parmer, Vernon Hills, IL, USA) and centrifuged for $5 \mathrm{~min}$ at $4^{\circ} \mathrm{C}$. Aliquots of supernatant were subjected to protein assay with Coomassie Plus assay reagent (Nanjing Jiancheng Bioengineering Institute) and ALP activity measurement using an ALP assay kit (Nanjing Jiancheng Bioengineering Institute). The enzyme activity was normalized against the protein concentration and expressed as $\mathrm{U} / \mathrm{g}$ protein. Furthermore, BMSCs were rinsed three times with PBS, fixed with formalin for $10 \mathrm{~min}$ and washed three times with PBS after two weeks of incubation. Cells were then stained 
with the ALP staining kit (Nanjing Jiancheng Bioengineering Institute). After three weeks of incubation, von Kossa staining was performed to detect calcium deposits. BMSCs were covered with $2 \%$ silver nitrate solution and exposed to ultraviolet light for $60 \mathrm{~min}$. Cells were then washed thoroughly with PBS and treated with $5 \%$ sodium thiosulfate for $2 \mathrm{~min}$. Cells were washed thrice with PBS and calcium deposits were observed using microscopy.

Adipogenic differentiation assays. P3 cells were seeded in growth medium at $2 \times 10^{4}$ cells $/ \mathrm{cm}^{2}$ in six-well tissue culture plates. At $100 \%$ confluence, the growth medium was replaced with OriCell SD rat mesenchymal stem cell adipogenic differentiation medium (Cyagen Biosciences, Inc.). After three days of incubation, the adipogenic differentiation media were replaced with adipogenic maintenance medium (Cyagen Biosciences, Inc.) for $24 \mathrm{~h}$. Three cycles of differentiation and maintenance medium replacement were performed. Following seven days of maintenance culture, cells were rinsed three times with PBS and covered with Oil Red O staining for $10 \mathrm{~min}$ at room temperature. Lipid droplets in the cells were observed using microscopy.

Quantitative polymerase chain reaction analysis ( $q P C R)$. Total RNA was isolated from individual cell layers at different passages after seven days of further incubation using TRIzol ${ }^{\circledR}$ (Invitrogen Life Technologies) following the manufacturer's instructions. Reverse transcription of mRNA (1 $\mu \mathrm{g})$ was performed using a Reverse Transcription System kit (Toyobo, Osaka, Japan). qPCR was performed using SYBR ${ }^{\circledR}$-Green Real Time PCR Master Mix (QPK-201; Toyobo). The expression of the following genes was examined: Basic fibroblast growth factor (bFGF), sonic hedgehog (Shh), core binding factor a1 (Cbfa1), osteocalcin (OC) and ALP. GAPDH was used as the control. Primer sequences used are listed in Table I. qPCR was performed for $2 \mathrm{~min}$ at $50^{\circ} \mathrm{C}$, then for $10 \mathrm{~min}$ at $95^{\circ} \mathrm{C}$. qPCR was subsequently performed for osteogenic gene expression in several passages followed by 40 amplification cycles (15 sec at $95^{\circ} \mathrm{C}, 60 \mathrm{sec}$ at $60^{\circ} \mathrm{C}$ ). Following the last cycle, a melt-curve was generated. Each PCR experiment was processed in triplicate. A melting curve analysis was performed to demonstrate the specificity of each PCR product as a single peak. The comparative threshold cycle method was used to evaluate the differences in gene expression.

Statistical analysis. Experimental data are reported as the mean \pm standard deviation. Histomorphometric data were assessed via analysis of variance (ANOVA) tests, with pairwise comparisons made by the Least Significant Difference procedure. A $\mathrm{P}<0.05$ was considered to indicate a statistically significant difference between values.

\section{Results}

Growth characteristics of BMSCs. Adherent, monoptychial cells were observed in the culture dishes after $24 \mathrm{~h}$ of incubation. At day 7, cells exhibited a fibroblast shape with a unique vortex arrangement at $90 \%$ confluence (Fig. 1A). These cells were designated P0 cells. BMSC cultures were homogenous populations, even following subculture for five passages or more. The growth of BMSCs was characterized at P3. The
Table I. Specific primers used for quantitative polymerase chain reaction.

\begin{tabular}{ll}
\hline Gene & \multicolumn{1}{c}{ Primers } \\
\hline bFGF & F: 5'-GGACGGCTGCTGGCTTCTAA-3' \\
& R: 5'-CCAGTTCGTTTCAGTGCCACATAC-3' \\
Shh & F: 5'-ATGAACGGACCTTCAAGAGCCTTA-3' \\
& R: 5'-AGCAGGTTGCTTGGCCTCA-3' \\
Cbfa1 & F: 5'-TGCTTCATTCGCCTCACAAA-3' \\
& R: 5'-TGCTGTCCTCCTGGAGAAAGTT-3' \\
OC & F: 5'-AGGACCCTCTCTCTGCTCAC-3' \\
& R: 5'-AACGGTGGTGCCATAGATGC-3' \\
ALP & F: 5'-TCCATGGTGGATTATGCTCA-3' \\
& R: 5'-TTCTGTTCCTGCTCGAGGTT-3' \\
GAPDH & F: 5'-TGAACGGGAAGCTCACTGG-3' \\
& R: 5'-TCCACCACCCTGTTGCTGTA-3' \\
\hline
\end{tabular}

F, forward; R, reverse; bFGF, basic fibroblast growth factor; Shh, sonic hedgehog; Cbfa1, core binding factor a1; OC, osteocalcin; ALP, alkaline phosphatase.

viability of BMSCs was detected from days 0 to 7 . The cell proliferation curve was similar to an S-shape curve, with the rate of proliferation peaking at day 2 and then plateauing 6 days later. The cells exhibited a logarithmic growth from days 2 to 5 (Fig. 1B). P3 cells were subjected to cell cycle analysis. Cell counts and cell cycle phase distributions were assessed using flow cytometry. Almost $90 \%$ of cells were in G0/G1 phase, $5.0 \%$ were in $\mathrm{G} 2$ phase and $5.7 \%$ in S phase. Most of the cells were in a quiescent state and only a small percentage of cells were in the active proliferation period, which was in accordance with the characteristics of stem cells (Fig. 1C).

Phenotypic characterization of BMSCs. To identify the BMSCs, flow cytometric analysis was used to detect the surface marker Ags. From the flow cytometric analysis, the cells of the P3 group were used to detect the expression of CD45 and CD29. Fig. 2A shows that $99.7 \%$ of cells expressed CD29, but did not appear to express CD45, a pan-hematopoietic marker. These cells were selected as the group P2 to detect the expression of CD90 and CD29. Furthermore, the results showed that 98.4\% of cells expressed CD29 and CD90 (Fig. 2A). A triple immunofluorescence technique was performed to validate the results of the flow cytometric analysis. As shown in Fig. 2B, the results indicated a strong staining of the cells by monoclonal antibodies directed to CD29 and CD90. Confirmation of the non-hematopoietic nature of the BMSCs was indicated by the absence of staining for CD45.

Multipotent differentiation potential of BMSCs. ALP activity of the supernatant medium and BMSCs was determined on days 3, 5, 7, 9, 11 and 13 of culture. ALP activity in BMSCs with induced osteogenicity was observed to be higher in comparison with that in the negative controls from day 5 (Fig. 3A). As indicated by the ANOVA, the ALP activity in BMSCs with induced osteogenicity was statistically higher 
A

$72 \mathrm{~h}$

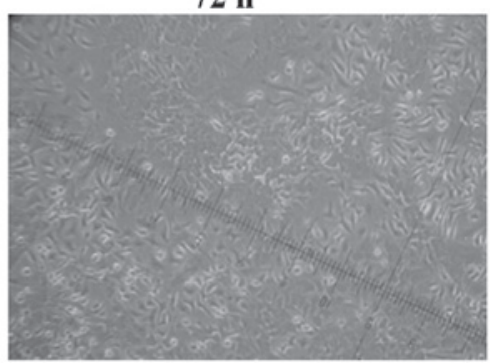

B

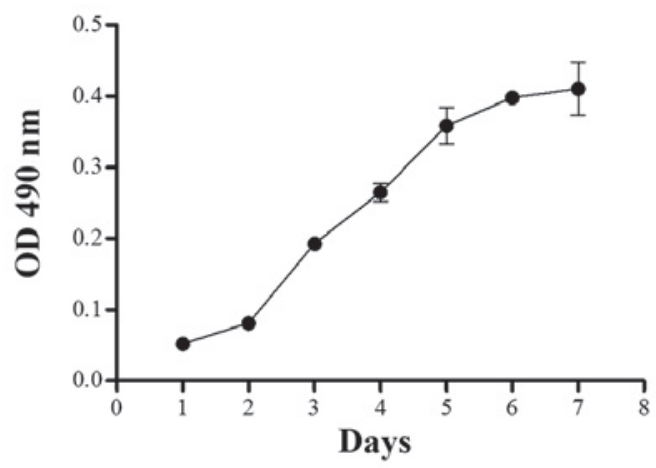

P1

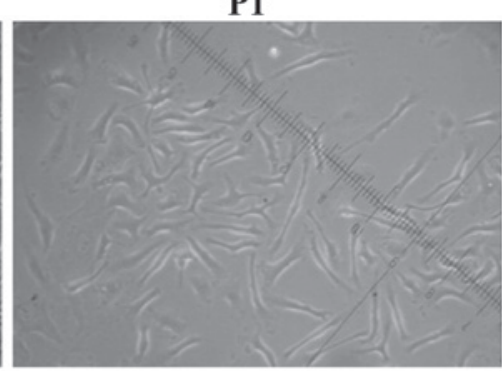

C

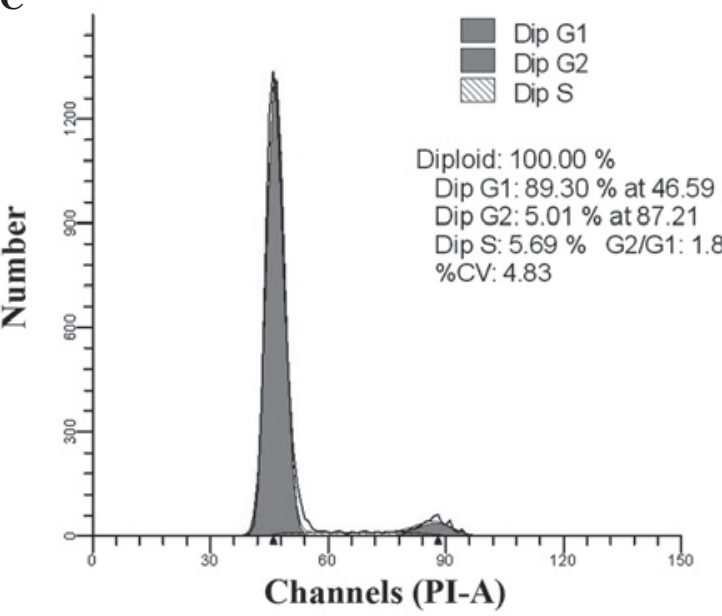

P3

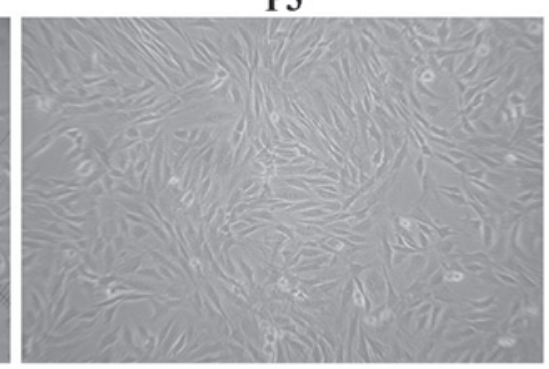

Dip G1 $89.30 \%$ at 46.59

Dip G2:5.01\% at 87.21

Dip S: $5.69 \%$ G2/G1: 1.87

(1)

Figure 1. (A) Appearance of the cultured BMSCs (magnification, $\mathrm{x} 40$ ). (B) Cell viability of BMSCs assessed using the MTT assay. (C) Cell cycle distribution of BMSCs assessed using flow cytometry. BMSCs, bone marrow-derived mesenchymal stem cells; P, passage; PI, propidium iodide; OD, optical density.

A

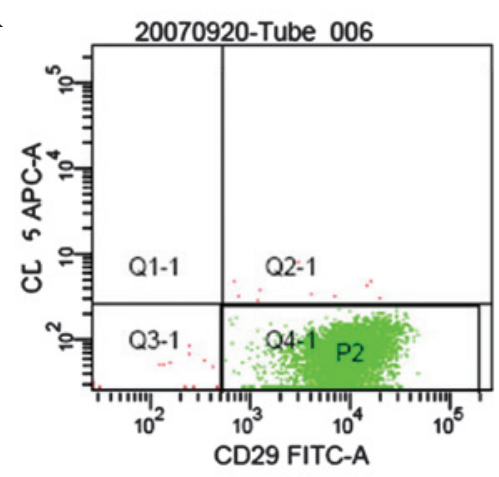

Lightscope

B

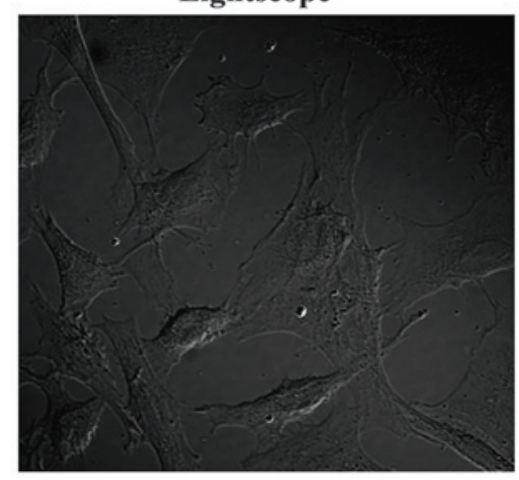



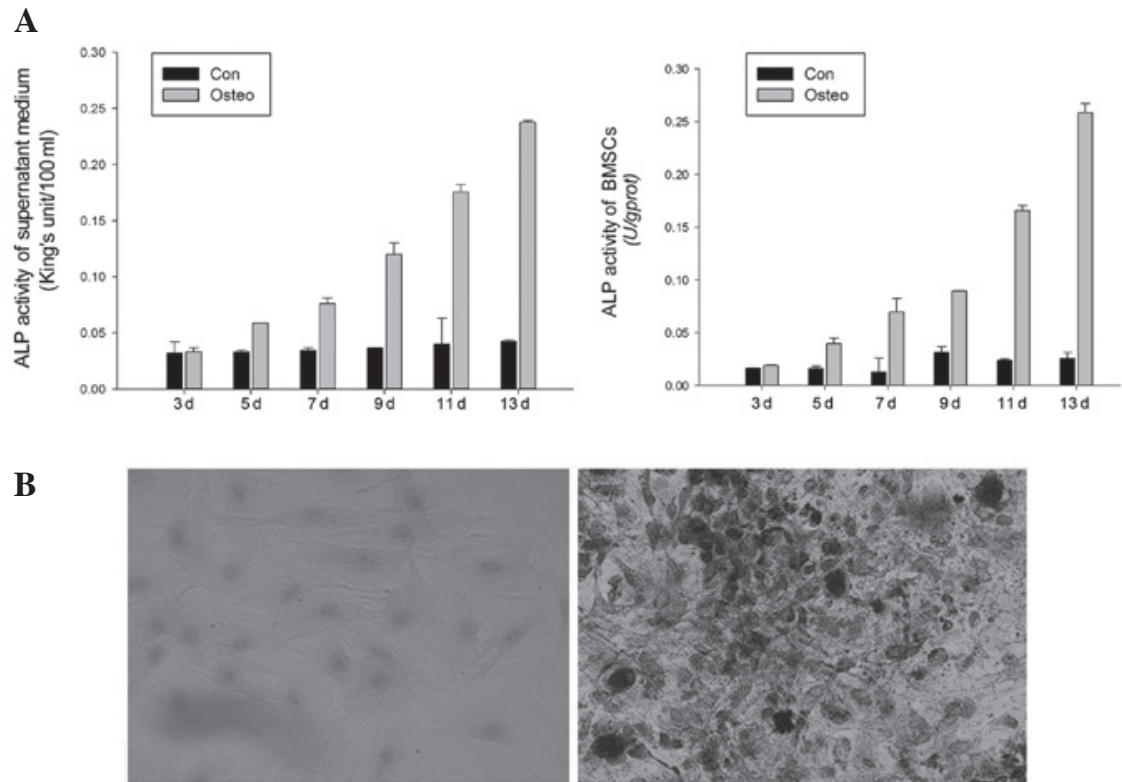

C
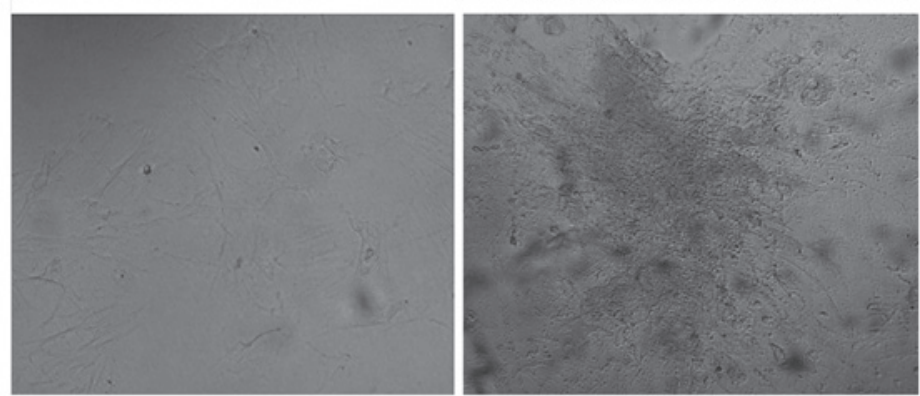

D

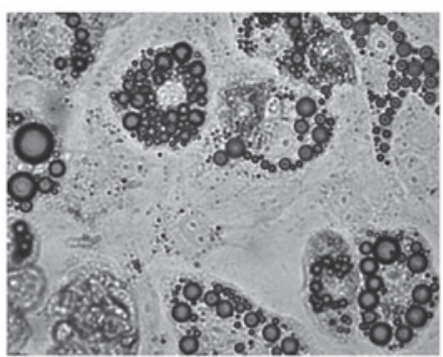

Figure 3. (A) ALP activity in supernatant medium and BMSCs. (B) ALP staining of BMSCs. Black granular material within the cytoplasm indicates ALP activity (magnification, x100; left image shows the control group). (C) Von Kossa staining of BMSCs (magnification, x40; left image shows the control group). (D) Oil Red O staining of BMSCs (magnification, x200). ALP, alkaline phosphatase; BMSCs, bone marrow-derived mesenchymal stem cells; Con, control; Osteo, osteogenesis-induced.

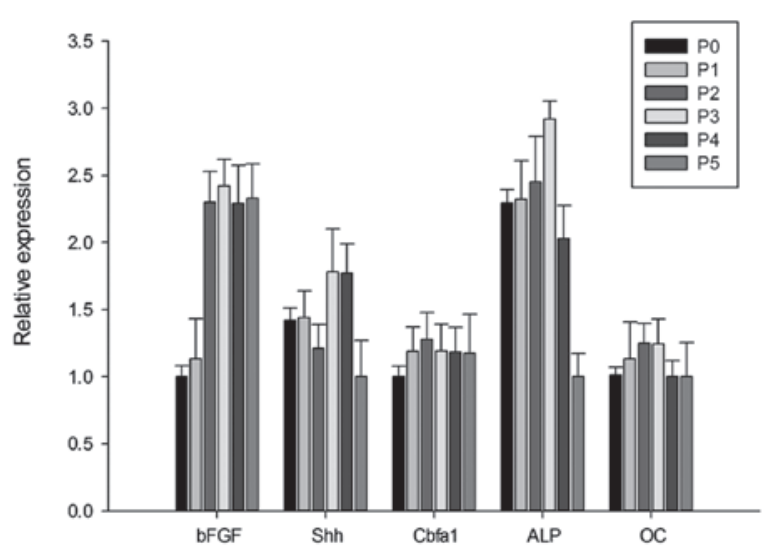

Figure 4. Osteogenic marker expression in bone marrow-derived mesenchymal stem cells as determined by quantitative polymerase chain reaction. bFGF, basic fibroblast growth factor; Shh, sonic hedgehog; Cbfa1, core binding factor a1; OC, osteocalcin; ALP, alkaline phosphatase; P, passage. than that of the negative controls on days 11 and 13. ALP staining was performed on day 14 (Fig. 3B), and the von Kossa staining was performed on day 21 (Fig. 3C). BMSCs cultured in the normal medium did not stain for ALP and von Kossa. However, BMSCs cultured in osteogenic medium stained positive for ALP and von Kossa. P3 cells were subjected to induction of adipogenic differentiation for two weeks. Red lipid droplets in the induced cells were observed by microscopy with Oil Red O staining (Fig. 3D).

Gene expression of BMSCs. The expression of osteoblast marker mRNA in BMSCs at different passages was assessed using qPCR. Endogenous gene expression of bFGF, Shh, Cbfa1 and ALP in BMSCs was observed at all passages (Fig. 4). The expression of bFGF increased progressively from $\mathrm{P} 0$ to $\mathrm{P} 3$. However, a parabola tendency was observed in the expression 
of Shh, Cbfal, ALP and OC. There were no statistical differences among the groups.

\section{Discussion}

According to the International Society for Cellular Therapy (11), cells that are isolated from bone marrow and other tissues and that exhibit plastic-adherent properties should be referred to as 'multipotent mesenchymal stromal cells' (MSCs). These cells have typically been labeled as mesenchymal stem cells (12). There are various methods to isolate and expand MSCs, including density gradient isolation (13), immunomagnetic isolation (14), flow cytometry separating (15) and plastic-adherent culture methods (16). Although higher purity BMSCs can be obtained using these methods, complex procedures and abundant bone marrow increase the difficulty of surgery and risk of contamination.

In the present study, adherent monoptychial cells were observed in the culture dishes after $24 \mathrm{~h}$ of incubation. The cells exhibited a logarithmic growth from days 2 to 5 , and $\sim 90 \%$ of cells were in G0/G1 phase. Most of the cells were in a quiescent stage, which was in accordance with the characteristics of stem cells.

In a previous study it was shown that the in vitro propagation of BMSCs markedly decreased their homing to bone marrow (17). The BMSCs of passages P0 to P5 were perfect and viable in the present study. Endogenous gene expression of bFGF, Shh, Cbfal and ALP was observed in BMSCs at all passages. In addition, the maximum expression of these osteoblast marker genes was detected at P3.

The biological property that most uniquely identifies BMSCs is their capacity for trilineage mesenchymal differentiation. The BMSCs at P3 were selected for induction of osteogenic and adipogenic differentiation. ALP activity in BMSCs with induced osteogenic differentiation was observed to be higher in comparison with that in the negative controls from day 5 , and was visibly increased from days 5 to 13 . ALP is an early marker of osteogenesis and, therefore, the increased ALP activity indicated that BMSCs were differentiated into osteogenic cells. Von Kossa staining was used to quantify mineralization in BMSCs with induced osteogenic differentiation. Mineralized nodules were observed at three weeks. In addition, red lipid droplets in adipogenic-induced cells were observed by microscopy following Oil Red O staining. This positive result demonstrated that the BMSCs were differentiated into adipose cells.

The analysis of surface Ag expression enables cell populations to be rapidly identified. This technique has been extensively used in immunology and hematology (18). To identify the BMSCs in the present study, flow cytometric analysis and a triple immunofluorescence technique were used to detect the surface marker Ags. The flow cytometric analysis revealed that $98.4 \%$ of cells expressed CD29 and CD90, but did not appear to express CD45. CD29 is the surface marker of mesenchymal cells, and CD90 is the surface marker of stem cells. However, CD45 is a pan-hematopoietic marker. Triple immunofluorescence analysis showed a strong staining for CD29 and CD90 and no staining for CD45.

In conclusion, in the present study, the BMSCs isolated from the bone marrow of rats were cultured and analyzed.
This fibroblast-like clone possesses the characteristics of stem cells. The cells at P3 exhibited the characteristics of mesenchymal stem cells and were able to differentiate into osteoblasts and adipocytes. BMSCs with these identified characteristics may thus be used as seed cells in bone tissue engineering.

\section{Acknowledgements}

The present study was supported by a grant from the Innovation Research Fund of Huazhong University of Science and Technology (no. 2013QN204).

\section{References}

1. Logeart-Avramoglou D, Anagnostou F, Bizios R and Petite H: Engineering bone: challenges and obstacles. J Cell Mol Med 9: 72-84, 2005 .

2. Toquet J, Rohanizadeh R, Guicheux J, et al: Osteogenic potential in vitro of human bone marrow cells cultured on macroporous biphasic calcium phosphate ceramic. J Biomed Mater Res 44: 98-108, 1999.

3. Rochet N, Loubat A, Laugier JP, et al: Modification of gene expression induced in human osteogenic and osteosarcoma cells by culture on a biphasic calcium phosphate bone substitute. Bone 32: 602-610, 2003.

4. Kruyt MC, Dhert WJ, Yuan H, et al: Bone tissue engineering in a critical size defect compared to ectopic implantations in the goat. J Orthop Res 22: 544-551, 2004.

5. Pittenger MF, Mackay AM, Beck SC, et al: Multilineage potential of adult human mesenchymal stem cells. Science 284: 143-147, 1999.

6. Ohgushi H, Dohi Y, Katuda T, Tamai S, Tabata S and Suwa Y: In vitro bone formation by rat marrow cell culture. J Biomed Mater Res 32: 333-340, 1996.

7. Kadiyala S, Young RG, Thiede MA and Bruder SP: Culture expanded canine mesenchymal stem cells possess osteochondrogenic potential in vivo and in vitro. Cell Transplant 6: 125-134, 1997.

8. Johnstone B, Hering TM, Caplan AI, Goldberg VM and Yoo JU: In vitro chondrogenesis of bone marrow-derived mesenchymal progenitor cells. Exp Cell Res 238: 265-272, 1998.

9. Bennett JH, Joyner CJ, Triffitt JT and Owen ME: Adipocytic cells cultured from marrow have osteogenic potential. J Cell Sci 99: 131-139, 1991.

10. Zuk PA, Zhu M, Mizuno H, et al: Multilineage cells from human adipose tissue: implications for cell-based therapies. Tissue Eng 7: 211-228, 2001.

11. Horwitz EM, Le Blanc K, Dominici M, et al; International Society for Cellular Therapy: Clarification of the nomenclature for MSC: The International Society for Cellular Therapy position statement. Cytotherapy 7: 393-395, 2005.

12. Caplan AI: Mesenchymal stem cells. J Orthop Res 9: 641-650, 1991.

13. Lisignoli G, Remiddi G, Cattini L, et al: An elevated number of differentiated osteoblast colonies can be obtained from rat bone marrow stromal cells using a gradient isolation procedure. Connect Tissue Res 42: 49-58, 2001.

14. Encina NR, Billotte WG and Hofmann MC: Immunomagnetic isolation of osteoprogenitors from human bone marrow stroma. Lab Invest 79: 449-457, 1999.

15. Zohar R, Sodek J and McCulloch CA: Characterization of stromal progenitor cells enriched by flow cytometry. Blood 90: 3471-3481, 1997.

16. Zhang B, Wang F, Deng L, et al: Isolating and culturing rat marrow mesenchymal stem cells and studying their phenotypical and functional properties. Sichuan Da Xue Xue Bao Yi Xue Ban 34: 738-741, 2003 (In Chinese).

17. Rombouts WJ and Ploemacher RE: Primary murine MSC show highly efficient homing to the bone marrow but lose homing ability following culture. Leukemia 17: 160-170, 2003.

18. Dominici M, Le Blanc K, Mueller I, et al: Minimal criteria for defining multipotent mesenchymal stromal cells. The International Society for Cellular Therapy position statement. Cytotherapy 8: 315-317, 2006. 